\title{
The Role of Repetitive Negative Thoughts in the Vulnerability for Emotional Problems in Non-Clinical Children
}

\author{
Suzanne Broeren - Peter Muris - Samantha Bouwmeester • \\ Kristiaan B. van der Heijden • Annemieke Abee
}

Published online: 26 May 2010

(C) The Author(s) 2010. This article is published with open access at Springerlink.com

\begin{abstract}
The current study examined the role of repetitive negative thoughts in the vulnerability for emotional problems in non-clinical children aged $8-13$ years $(N=158)$. Children completed self-report questionnaires for assessing (1) neuroticism and behavioral inhibition as indicators of general vulnerability (2) worry and rumination which are two important manifestations of repetitive negative thoughts, and (3) emotional problems (i.e., anxiety, depression, and sleep difficulties). Results demonstrated that there were positive correlations between measures of general vulnerability, repetitive negative thoughts, and emotional problems. Further, support was found for a model in which worry and rumination acted as partial mediators in the relation between neuroticism and symptoms of anxiety and depression. In the case of sleep difficulties, no evidence was obtained for such a mediation model. In fact, data suggested that sleeping difficulties are better conceived as an epiphenomenon of high symptom levels of anxiety and depression or as a risk factor for the development of other types of psychopathology. Finally, besides neuroticism, the temperamental trait of behavioral inhibition appeared to play a unique direct role in the model predicting anxiety symptoms but not in the models predicting depressive symptoms or sleep difficulties. To conclude, the current findings seem to indicate that worry and rumination contribute to children's vulnerability for anxiety and depression.
\end{abstract}

\footnotetext{
S. Broeren $(\bowtie) \cdot$ P. Muris $\cdot$ S. Bouwmeester $\cdot$ A. Abee Institute of Psychology, Erasmus University Rotterdam, Burgemeester Oudlaan 50, Suite T13-08, P.O. Box 1738, 3000 DR Rotterdam, The Netherlands

e-mail: broeren@fsw.eur.nl

K. B. van der Heijden

Centre for Child and Family Studies, Leiden University, Leiden,

The Netherlands
}

Keywords Worry · Rumination · Anxiety - Depression · Sleep problems $\cdot$ Vulnerability $\cdot$ Children

\section{Introduction}

Emotional problems such as anxiety and depression are common among children. For example, Costello et al. (2003) estimated in their longitudinal community study among children that by the age of $16,7.7 \%$ of all children had met the diagnostic criteria for an anxiety disorder, whereas $7.3 \%$ had met the diagnostic criteria for any depressive disorder. These disorders are associated with significant impairment in social, emotional, and school functioning (Fleming and Offord 1990; Strauss et al. 1987). Sleep problems have been found to frequently co-occur with symptoms of anxiety and depression (e.g., Ryan et al. 1987) and are even part of the DSM-IV criteria of major depressive disorder (MDD), post traumatic stress disorder and generalized anxiety disorder (GAD; American Psychiatric Association 2000). In children diagnosed with an anxiety disorder for instance, Alfano et al. (2007) noted that $88 \%$ reported at least one sleep-related difficulty (e.g., insomnia, sleep initiation and maintenance problems, nightmares). In a similar vein, Liu et al. (2007) observed that approximately two out of three children with MDD reported sleep difficulties. These high comorbidity figures have made some authors to propose that sleep difficulties should be seen as a direct consequence of other psychiatric problems rather than as a primary psychopathology on its own (Lichstein 2006). However, research in adults seems to indicate that, contrary to the widely held belief that sleep difficulties can best be seen as "secondary psychopathology", insomnia can be better viewed as a primary 
psychopathology in that it often develops before its comorbid disorders such as MDD and anxiety disorders, and hence should be regarded as a risk factor for the development of these comorbid psychiatric disorders (Harvey 2001a, b). Nonetheless, sleep disturbances can also have a large negative impact on the daily functioning of non-clinical children and adolescents, with disturbed sleep leading to significant daytime sleepiness, which in its turn is associated with behavioral difficulties, decreased positive mood, and impairments in academic performance and attention (Fallone et al. 2002). Therefore, it is not only important to investigate factors involved in the etiology and maintenance of anxiety and depression, but also factors involved in the pathogenesis of sleep difficulties.

Repetitive negative thoughts are generally viewed as a specific cognitive vulnerability factor that seems to be involved in the etiology and maintenance of emotional problems. Two prominent examples of repetitive negative thoughts are worry and rumination. Worry can be defined as a relatively uncontrollable chain of thoughts about the possible negative outcome of future events (Borkovec et al. 1983), whereas rumination can be defined as a tendency to repeatedly think about one's feelings and problems (Nolen-Hoeksema 1998, 2000; Nolen-Hoeksema et al. 2008). The concepts of worry and rumination seem to show considerable overlap (McLaughlin et al. 2006; NolenHoeksema et al. 2008): Both constructs seem to tap repetitive, negative thoughts that are focused on the self (Segerstrom et al. 2000), share an over-general style of thinking (Watkins and Teasdale 2001), and are associated with cognitive inflexibility and poor problem-solving (e.g., Watkins and Moulds 2005; Davey 1994). However, both phenomena differ in their orientation and the type of threat they focus on: while worry tends to be more future-oriented and focuses on events that might occur, but have not occurred yet, rumination involves thinking about past events and wondering about the causes and meaning of those events. Additionally, various studies have shown that worry and rumination are related but statistically distinguishable constructs (e.g., Fresco et al. 2002; Hong 2007).

There is increasing evidence that both worry and rumination are already discernable in childhood and are also possibly linked to symptoms of anxiety and depression in youths. For example, in a non-clinical sample of 7- to 12-year-old children, Silverman et al. (1995) showed that worry was significantly correlated with anxiety. Further, it was found that various worry parameters were able to successfully discriminate between high and low anxious children, thereby providing further support for the intimate link between worry and anxiety. Comparable results have been documented in research investigating the relation between rumination and childhood depression. Exemplary is a study by Abela et al. (2002), who explored the relation between rumination and depressive symptoms in young people and demonstrated that rumination scores prospectively predicted increases in depressive symptoms over a 6-week period. A recent metaanalytic review by Rood et al. (2009) which assessed the cross-sectional and longitudinal relations between emotionfocused rumination and depressive symptoms also indicated that rumination was associated with concurrent and future levels of depression in children and adolescents.

However, while most researchers tend to relate worry exclusively to anxiety and rumination solely to depression, there is now an increasing amount of research data showing that worry is also related to depression, whereas rumination is also connected to anxiety, and this is not only the case for adults (e.g., Starcevic 1995) but also for youths. For instance, Muris et al. (2004) conducted a study on the relations between worry, rumination, anxiety and depression in a sample of young non-clinical adolescents. Results indicated that worry and rumination displayed different features, but were nevertheless substantially related to each other. In addition, it was found that both types of repetitive negative thoughts were significantly related to anxiety as well as depression, with worry being the most convincing correlate of these emotional symptoms (see also Muris et al. 2009b).

While the link between worry and rumination on the one hand, and emotional problems on the other hand gets increasing research attention, so far, no studies have been conducted to examine the link between these repetitive negative thoughts and sleep difficulties in children. Nevertheless, it seems intuitively plausible that repetitive negative thought processes are involved in such problems and there is tentative evidence from a study in adults to support this notion. Evidence is found that rumination is related to lower (subjective) sleep quality (Kirkegaard Thomsen et al. 2003; Guastella and Moulds 2007) and sleep difficulties (Carney et al. 2006), whereas pre-sleep worries seem to be associated with insomnia (Harvey 2001a, b) and prolonged self-reported and objective sleep latencies (Harvey 2000; Wicklow and Espie 2000).

Recently, Muris et al. (2009b) have formulated a theoretical model on the role of repetitive negative thoughts in emotional problems which is based on the notion of Segerstrom et al. (2000) that worry and rumination seem to represent one underlying cognitive factor and the observation that both constructs are substantially linked to neuroticism. In this model, worry and rumination are both seen as cognitive factors that follow from the general vulnerability factor of neuroticism (Eysenck 1967), which predisposes individuals to develop high symptom levels of anxiety and depression. Indeed, Muris et al. (2009b) obtained support for this model in a sample of undergraduate students as they demonstrated that the cognitive factors of worry and rumination acted as mediators in the relationship between neuroticism and symptoms of anxiety 
and depression. However, it has to be kept in mind that the data of this study were correlational in nature, so no causeeffect relations could be established, although from a theoretical point of view this chain of relationships among these variables seems plausible (e.g., Norton et al. 2005; Sexton et al. 2003). Furthermore, Roelofs et al. (2008) also found support for this model in a sample of clinically depressed individuals. The results of their study showed that worry and rumination both acted as mediators in the relation between neuroticism and symptoms of anxiety and depression. However, when worry and rumination were entered simultaneously in a mediation model, only rumination was found to mediate the relation between neuroticism and both types of emotional symptoms.

Further, while it can be assumed that neuroticism serves as a vulnerability factor for childhood anxiety symptoms, behavioral inhibition, which can be defined as the tendency to withdraw from new situations (Kagan 1994), may even be more important when studying etiological models of this type of psychopathology (Fox et al. 2005). Behavioral inhibition is sometimes seen as the perceptible (behavioral) manifestation of temperament or personality traits such as emotionality, negative affectivity, or neuroticism (e.g., Craske 1997; Lonigan and Phillips 2001) and there is indeed empirical evidence for a link between behavioral inhibition and neuroticism (Muris and Dietvorst 2006; Muris et al. 2009a). However, other research shows that behavioral inhibition might better be seen as the combination of high neuroticism and low extraversion or regulative traits (Muris and Dietvorst 2006; Van Brakel and Muris 2006). Children scoring high on behavioral inhibition seem to be at heightened risk for developing an anxiety disorder (e.g., Biederman et al. 1993, 2001). So far, research has emphasized that behavioral inhibition is a risk factor for developing childhood anxiety disorders. However, there are two studies by Muris and colleagues who also link behavioral inhibition to depressive symptoms (Muris et al. 1999, 2001). These studies showed that high behaviorally inhibited children not only displayed heightened levels of anxiety but also elevated levels of depressive symptoms. The link between behavioral inhibition and sleep problems has not been studied yet.

Although the role of worry and rumination in the etiology of emotional problems is increasingly investigated in youths (Muris et al. 2004, 2009b), so far no study can be found that examined the mediation model "neuroticism $\rightarrow$ worry/rumination $\rightarrow$ anxiety/depression" in a child population, notwithstanding the fact that research has shown that repetitive negative thoughts are already present in children. Better insight into the pathogenic mechanisms of emotional problems might contribute to the development of more optimal psychological interventions. For instance, if rumination and worry play an important role in the pathogenesis of anxiety and depression, it would support the importance of cognitive treatment approaches directed at correction of these cognitive factors. With this in mind the present study was conducted. Children aged 8-12 years $(N=158)$ completed a survey containing scales for measuring neuroticism, worry and rumination, and symptoms of emotional problems. Besides a correlational analysis of the relations among these variables and a test of the hypothesized mediation model, a number of additional issues were explored. To begin with, we not only included measures of anxiety and depression as symptom variables, but also an index of children's sleep difficulties, although we are well aware of the possible overlap between sleep problems and symptoms of MDD and GAD. This provided the opportunity for a first exploration of the links between worry and rumination and sleep difficulties in a population of youths, and to test whether the mediation model described earlier also applies to this type of emotional problem. Further, while it can be assumed that neuroticism serves as a vulnerability factor for childhood anxiety and depressive symptoms, behavioral inhibition might also play an important role in the etiology of psychopathology in children, especially anxiety symptoms pathology (Fox et al. 2005). Therefore, it would be interesting to examine whether this temperament variable accounts for unique variance (even when controlling for neuroticism) in the prediction of repetitive negative thought and symptoms of psychopathology, and anxiety symptoms in particular.

We hypothesized that in this sample of non-clinical children, (a) neuroticism would be positively associated with symptoms of anxiety and depression, and sleep problems, (b) neuroticism would also be positively associated with worry and rumination, (c) both worry and rumination would be positively associated with symptoms of anxiety, depression, and sleep problems, and (d) the associations between neuroticism and the various types of psychopathology would be reduced (partial mediation) or eliminated (full mediation) when controlling for the mediating variables of worry and rumination. Further, we predicted that behavioral inhibition would remain a significant predictor of anxiety symptoms when controlling for neuroticism, while we explored whether this temperament characteristic has unique predictive value for depressive symptoms or sleep difficulties.

\section{Method}

Participants

One-hundred-and-fifty-eight children ( 84 boys and 74 girls) participated in this study. The mean age of the children was 10.73 years $(\mathrm{SD}=1.01$; range $8-13)$. The 
majority of the children were from original Dutch descent (98.10\%). Children were recruited from four primary schools in Zwijndrecht and Barendrecht, the Netherlands. Prior to the study parents were asked to fill in an informed consent form; $38 \%$ of the invited parents agreed to participate. This study was approved by the local ethical committee of the Institute of Psychology of Erasmus University Rotterdam.

\section{Assessment}

A subscale of the Big Five Questionnaire for Children (BFQ-C; Barbaranelli et al. 2003) was employed to measure the personality trait of neuroticism. Items such as "I get nervous for silly things" and "I am in a bad mood" have to be rated on a four-point Likert scale with $1=n o t$ true to $4=$ very true, and a total score can be computed by summing all items. Previous research has demonstrated that this subscale of the BFQ-C is reliable in terms of internal consistency (with Cronbach's alpha $=.83$ ) and possesses adequate validity as demonstrated by a positive correlation with its counterpart subscale of the Junior version of the Eysenck Personality Questionnaire (Eysenck and Eysenck 1985; see Muris et al. 2005a; $r=.71$ ).

Behavioral inhibition was measured with the Behavioral Inhibition Questionnaire for Children-Short Form (BIQC-SF; Bishop et al. 2003), which consists of 14 items assessing children's inhibited behaviors in social and nonsocial contexts (e.g., "I am very quiet with adult strangers", "I am cautious in activities that involve physical challenge"). Items are rated on a six-point scale ranging from $1=$ hardly ever to $6=$ almost always. A total BIQ$\mathrm{C}-\mathrm{SF}$ score can be calculated by summing across all items. The self-report version of the BIQ-C-SF has not been used in research before, but Edwards (2007) examined the psychometric properties of the parent version of this questionnaire (BIQ-SF) and documented good internal consistency (with a Cronbach's alpha of .89) and validity as indicated by a positive correlation with another measure of inhibited and shy behaviour (i.e., the Short Temperament Scale for Children; Prior et al. 1989; $r=.87$ ).

The Penn State Worry Questionnaire for Children (PSWQ-C; Chorpita et al. 1997) is a 14-item scale for measuring the tendency to engage in excessive, generalized, and uncontrollable worry. Items such as "Once I start worrying, I can't stop" and "I notice that I have been worrying about things" are rated on a four-point Likertscale with $1=$ not at all true and $4=$ always true. A PSWQ-C total score can be computed by summing across all items. The psychometric properties of the PSWQ-C are found to be adequate. That is, the questionnaire has been shown to possess adequate reliability in terms of internal consistency (with Cronbach's alphas in the .80 range) and temporal stability ( 1 week test-retest: $r=.92$; Chorpita et al. 1997). In addition, the PWSQ-C correlates positively with scores on anxiety questionnaires ( $r$ s between .44 and .71), which supports the validity of the scale.

Rumination was assessed with the Children's Response Style Scale (CRSS; Ziegert and Kistner 2002). This questionnaire contains 10 items for measuring the ruminative response styles of children when confronted with feelings of depression (e.g., "When I feel sad, I think back to other times I felt this way"). Items have to be scored on a fivepoint scale with $1=$ never and $5=$ always. Ziegert and Kistner (2002) have demonstrated that the rumination scale of the CRSS is reliable in terms of internal consistency (Cronbach's $\alpha=.81$ ) and test-retest stability (3-week period: $r=.69$ ). Furthermore, CRSS rumination scores were positively associated with depression $(r=.25)$ and an alternative self-report measure of the ruminative response style $(r=.39)$, which provides at least some support for the criterion and construct validity.

The Revised Children's Anxiety and Depression Scale (RCADS; Chorpita et al. 2000) measures symptoms of DSM-IV-defined anxiety disorders and major depression. The shortened version of this scale (Muris et al. 2002) consists of 25 items (e.g., "I am afraid to talk in front of class" and "I feel that nothing is much fun anymore") that have to be rated on a 4-point scale with $1=$ never to $4=$ always. Total RCADS anxiety and depression scores can be obtained by summing across relevant items. The psychometric properties of the short version of the RCADS are shown to be adequate (Muris et al. 2002). That is, the RCADS subscales display sufficient to good internal consistency (with Cronbach's alphas between .71 and .83), except from the 5-item depression subscale which shows moderate internal consistency (Cronbach's alpha $=.65$ ), and all scales show adequate test-retest stability (1 week period: $r$ s between .79 and .87). Further, positive associations have been found between various RCADS scales and other measures of childhood anxiety and depression (Chorpita et al. 2000; Muris et al. 2002; $r$ s between .27 and .78).

The Sleep Self Report (SSR; Owens et al. 2000) is a 26-item self-report questionnaire for assessing various sleep difficulties in children, namely problematic sleep behavior (e.g., "Do you wake up at night when your parents think you are asleep?"), sleep onset problems (e.g., "Do you fall asleep in about 20 min?"), and daytime sleepiness (e.g., "Do you feel sleepy during the day?"). After recoding reversed items, a total score can be computed by summing all relevant items. So far, few studies have been carried out to investigate the psychometric properties of the SSR. Research by Owens et al. (2000) has shown that children's SSR scores are positively associated with scores on a parent measure of sleeping difficulties ( $r$ s between .35 and 1.00). 
In the present study, a SSR total scale was employed, which displayed good internal consistency (Cronbach's alpha $=.80$ ).

\section{Procedure}

Parents received an informed consent letter via their child's teacher. After obtaining informed consent from their parents, children were visited at school by a research assistant who asked them to complete a survey, which contained the BIQ-C-SF, BFQ-C, PSWQ-C, CRSS, RCADS, and SSR. During the administration of the questionnaires, the research assistant was present to answer questions if necessary and to ensure confidentiality and independent responding. Children were rewarded with a small present (e.g., booklet, pen) in return for their participation.

\section{Data Analysis}

Data were analyzed by means of correlational analyses and independent $t$-tests. To control for type I error, $p$-values were adjusted to the number of tests that were carried out for the main analyses. Furthermore, a number of multiple mediator models were tested. The hypothesized mediation models were estimated using regression analyses in combination with a bootstrapping procedure. The personality trait of neuroticism was used as the independent variable in the mediation models. In the case of anxiety symptoms, an additional model was tested in which not only neuroticism was included as an independent variable but also behavioral inhibition. Worry and rumination were the mediators in the model, whereas one type of psychopathology symptoms (i.e., anxiety, depression, or sleep difficulties) was employed as the dependent variable. To reduce the chance of a type I error, alpha was here also adjusted to $.05 / 9$ (i.e., the total number of models tested in the mediation analyses). Furthermore, the regression weights and standard errors for the direct effects (i.e., between the independent and dependent variable) were estimated in the usual way, that is, by means of regression analysis. However, this was not possible for the indirect effects (i.e., the links between the independent variable and the mediators and between the mediators and the dependent variable), because these effects have to be calculated from the product of two direct effects and the distribution of this product can not assumed to be normal (Preacher and Hayes 2008). This non-normality is particularly problematic when the sample size is moderate, as was the case in the present study (i.e., $N=158$ ). In addition, it should be mentioned that the Sobel (1986) test, which is commonly used to examine indirect effects and mediation hypotheses, also requires a normal distribution of the variables involved. Because of the relatively small sample size and the intention to test the effects of two mediators simultaneously, it was not recommendable to employ the Sobel test. To explore the indirect effects and to test the mediation hypotheses, we employed Preacher and Hayes' (2008) bootstrapping procedure by means of a special macro that was programmed in SPSS. This macro was used to estimate a 95\% (bias corrected) confidence interval for the total and specific indirect effects of the mediators. When applicable, pairwise contrasts of indirect effects were used to test whether differences in the size of indirect effects were statistically significant.

\section{Results}

\section{General Findings}

Before addressing the main results of the study, a number of general findings will be reported. To begin with, as can be seen in Table 1, questionnaires were generally reliable in terms of internal consistency, with most Cronbach's alpha coefficients being $>.70$. Only the RCADS depression subscale displayed a lower internal consistency coefficient $($ Cronbach's alpha $=.58)$, which could have been due to the fact that only five items were included in this subscale that measured quite different symptoms of major depressive disorders (e.g., "I feel that nothing is much fun anymore" and "I feel like I don't want to move"). Further, significant gender differences were found for PSWQ-C worry, CRSS rumination, and RCADS anxiety symptoms. More precisely, girls displayed higher levels of worry $[t(128,84)=3.18$, $p<.01]$, rumination $[t(156)=2.29, p<.05]$, and anxiety symptoms $[t(135,07)=2.49, p<.05]$ as compared to boys (see Table 1). Finally, age was negatively associated with RCADS depression $(r=-.21, p<.01)$ and SSR Total sleep difficulties $(r=-.22, p<.01)$.

\section{Correlational Analysis}

Table 2 shows the partial correlations (while controlling for age and gender) between the variables measured in this study. First, the personality trait of neuroticism was strongly and positively correlated with the temperamental trait of behavioral inhibition $(r=.48, p<.001)$. Second, rumination and worry scores were also strongly and positively associated $(r=.57, p<.001)$. Third, substantial positive correlations were found between neuroticism and symptoms of anxiety $(r=.63, p<.001)$, depression $(r=.63, p<.001)$, and sleep difficulties $(r=.48$, $p<.001$ ), whereas behavioral inhibition was clearly related to symptoms of anxiety $(r=.53, p<.001)$. Fourth, positive associations were observed between neuroticism and behavioral inhibition, on the one hand, and the 
Table 1 Descriptive statistics (means, standard deviations, gender differences, and reliability coefficients) of the measures used in this study

\begin{tabular}{|c|c|c|c|c|}
\hline & $\begin{array}{l}\text { Total group } \\
(N=158)\end{array}$ & $\begin{array}{l}\text { Boys } \\
(n=84)\end{array}$ & $\begin{array}{l}\text { Girls } \\
(n=74)\end{array}$ & $\alpha$ \\
\hline BFQ-C neuroticism & $21.24(5.71)$ & $20.93(5.47)_{\mathrm{a}}$ & $21.59(5.97)_{\mathrm{a}}$ & .84 \\
\hline BIQ-C-SF behavioral inhibition & $33.12(13.03)$ & $32.95(13.13)_{\mathrm{a}}$ & $33.31(13.00)_{\mathrm{a}}$ & .89 \\
\hline PSWQ-C worry & $23.99(8.71)$ & $21.94(6.95)_{\mathrm{a}}$ & $26.32(9.89)_{\mathrm{b}}$ & .95 \\
\hline CRSS rumination & $24.67(8.63)$ & $23.21(8.01)_{\mathrm{a}}$ & $26.32(9.06)_{b}$ & .90 \\
\hline RCADS anxiety & $30.16(6.85)$ & $28.89(5.80)_{\mathrm{a}}$ & $31.61(7.66)_{\mathrm{b}}$ & .87 \\
\hline RCADS depression & $7.28(1.76)$ & $7.15(1.65)_{\mathrm{a}}$ & $7.43(1.89)_{\mathrm{a}}$ & .58 \\
\hline SSR total sleep difficulties & $33.43(6.61)$ & $34.94(6.63)_{\mathrm{a}}$ & $35.99(6.94)_{\mathrm{a}}$ & .80 \\
\hline
\end{tabular}

$B F Q-C$ Big Five Questionnaire for Children, BIQ-C-SF Behavioral Inhibition Questionnaire for Children-Short Form, $P S W Q-C$ Penn State Worry Questionnaire for Children, CRSS Children's Response Styles Scale, RCADS Revised Child Anxiety and Depression Scale, SSR Sleep Self Report. Means with different subscripts differ at $p<.05$

Table 2 Partial correlations between the different measures employed in this study for the total sample (controlling for age and gender) and for boys and girls separately (controlling for age)

\begin{tabular}{|c|c|c|c|c|c|c|c|c|c|c|c|c|}
\hline & (1) & (2) & (3) & (4) & (5) & (6) & $\begin{array}{l}\text { (1) Boys/ } \\
\text { girls }\end{array}$ & $\begin{array}{l}\text { (2) Boys/ } \\
\text { girls }\end{array}$ & $\begin{array}{l}\text { (3) Boys/ } \\
\text { girls }\end{array}$ & $\begin{array}{l}\text { (4) Boys/ } \\
\text { girls }\end{array}$ & $\begin{array}{l}(5) \text { Boys/ } \\
\text { girls }\end{array}$ & $\begin{array}{l}\text { (6) Boys/ } \\
\text { girls }\end{array}$ \\
\hline \multicolumn{13}{|l|}{ (1) BFQ-C neuroticism } \\
\hline $\begin{array}{l}\text { (2) BIQ-C-SF behavioral } \\
\text { inhibition }\end{array}$ & $.48^{*}$ & & & & & & $.24 / .71 *$ & & & & & \\
\hline (3) PSWQ-C worry & $.57 *$ & $.41 *$ & & & & & $.55 * / .61 *$ & $.30 * / .50 *$ & & & & \\
\hline (4) CRSS rumination & $.47 *$ & $.37 *$ & $.57 *$ & & & & $.36 * / .61 *$ & $.21 / .52 *$ & $.61 * / .59 *$ & & & \\
\hline (5) RCADS anxiety & $.63^{*}$ & $.53 *$ & $.71 *$ & $.62 *$ & & & $.51 * / .76 *$ & $.45 * / .60 *$ & $.80 * / .70 *$ & $.52 * / .74 *$ & & \\
\hline (6) RCADS depression & $.63^{*}$ & $.43 *$ & $.54 *$ & $.44 *$ & $.65^{*}$ & & $.53 * / .70 *$ & $.32 * / .53 *$ & $.48 * / .59 *$ & $.37 * / .52 *$ & $.57 * / .72 *$ & \\
\hline $\begin{array}{l}\text { (7) SSR total sleep } \\
\text { difficulties }\end{array}$ & $.48^{*}$ & $.30 *$ & $.36^{*}$ & $.27 *$ & $.51 *$ & $.51^{*}$ & $.30 * / .65 *$ & $.18 / .43^{*}$ & $.19 / 48 *$ & $.15 / .44 *$ & $.40 * / .62 *$ & $.29 * / .69 *$ \\
\hline
\end{tabular}

$N=158 ; n$ boys $=84, n$ girls $=74 . B F Q-C$ Big Five Questionnaire for Children, BIQ-C-SF Behavioral Inhibition Questionnaire for ChildrenShort Form, PSWQ-C Penn State Worry Questionnaire for Children, CRSS Children's Response Styles Scale, RCADS Revised Child Anxiety and Depression Scale, SSR Sleep Self Report. $* p<.01$

presumed mediators of worry and rumination on the other hand ( $r$ 's being between .37 and .57 , all $p$ 's $<.001$ ). Additionally, the mediators worry and rumination were also positively associated with the dependent variables (i.e., various types of emotional problems: anxiety, depression, and sleep difficulties; $r$ 's being between .27 and .71 , all $p$ 's $<.001$ ).

Table 2 also shows partial correlations (while controlling for age) for boys and girls separately. As can be seen, the same pattern of correlations as described above was observed, although in boys generally somewhat lower correlations among various concepts were observed than in girls.

\section{Mediation Analyses}

\section{Anxiety Symptoms}

The first model examined whether the relation between neuroticism and anxiety symptoms was mediated by worry and rumination. Figure 1 displays this hypothesized mediation model and its main results. A significant relation was found between neuroticism and anxiety symptoms $(B=.78, \mathrm{SE}=.07, p<.001)$. Neuroticism, worry and rumination together explained $65.51 \%$ of the variance in anxiety symptoms. Forty-five percent of this explained variance consisted of the direct relation between neuroticism and anxiety and this direct link appeared to be significant $(B=.35, \mathrm{SE}=.07, p<.001)$. The remaining

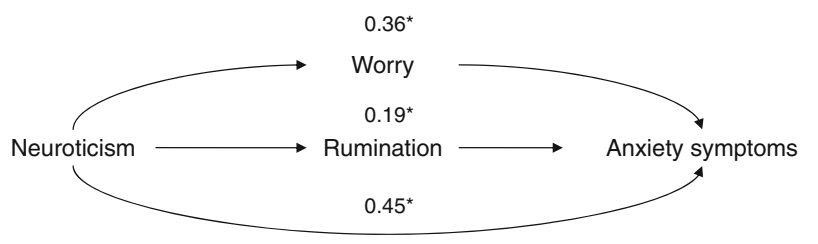

Fig. 1 Mediation effects of rumination and worry on the relation between neuroticism and anxiety symptoms. Note. Numbers in the figure are percentages of explained variance in anxiety symptoms. * Path significant at $p<.01$ 
$55 \%$ of the explained variance consisted of the total indirect effect through worry and rumination. The total indirect effect from neuroticism through worry and rumination to anxiety symptoms was significant [95\% CI (.29-.57)]. Additionally, the specific indirect effects via worry [95\% CI (.14-.42)] and rumination [95\% CI (.06-.26)] were also significant, and accounted for, respectively 36 and $19 \%$ of the variance. A pairwise contrast indicated that there was no significant difference in the size of these indirect effects [95\% CI (-.07-.32)], which means that worry and rumination did not made a different contribution to the model.

Given the high comorbidity between anxiety and depression, this analysis was also conducted including depressive symptoms as a covariate. Results were similar to the result of the previous analyses: there was still a significant relation between neuroticism and anxiety symptoms $(B=.48, \mathrm{SE}=.09, p<.001)$. Neuroticism, worry and rumination together explained $69 \%$ of the variance in anxiety symptoms (while controlling for depressive symptoms). Forty-five percent of this explained variance consisted of the direct relation between neuroticism and anxiety and this direct link appeared to be significant $(B=.22, \mathrm{SE}=.08, p<.01)$. The remaining $55 \%$ of the explained variance consisted of the total indirect effect through worry and rumination. The total indirect effect from neuroticism through worry and rumination to anxiety symptoms was significant [95\% CI (.14-.42)]. Additionally, the specific indirect effects via worry $[95 \%$ CI (.07-.31)] and rumination [95\% CI (.04-.18)] were also significant, and accounted for, respectively 36 and $19 \%$ of the variance. A pairwise contrast indicated that there was no significant difference in the size of these indirect effects [95\% CI (-.04-.22)], thus that worry and rumination did not have a significantly different contribution to the model. These findings indicate that (even when controlling for depressive symptoms) worry and rumination together indeed explained unique variance in anxiety symptoms over and above the personality trait of neuroticism, and that both variables acted as partial mediators in the relation between neuroticism and anxiety symptoms in children.

Analyses that included behavioral inhibition instead of neuroticism in the mediation model generally yielded similar findings. That is, the relation between behavioral inhibition and anxiety symptoms was partly mediated by worry and rumination. Additionally, a mediation model was tested in which the relation between behavioral inhibition and anxiety symptoms was examined while controlling for neuroticism to examine whether behavioral inhibition still had a significant effect when controlling for neuroticism. Behavioral inhibition, worry, and rumination together explained $65 \%$ of the variance in anxiety scores (while controlling for neuroticism). Of the total effect (while controlling for neuroticism), 67\% of the variance could be explained by the direct relation between behavioral inhibition and anxiety symptoms $(B=.10$, $\mathrm{SE}=.03, p<.001$ ), whereas the remaining $33 \%$ of the explained variance consisted of the indirect effect through worry (accounting for $20 \%$ of this explained variance) and rumination (accounting for 13\%). However, the total indirect effect was not significant [95\% CI $(-.00-.10)]$ and neither were the two specific indirect effects. These findings indicate that when controlling for neuroticism, the temperamental trait of behavioral inhibition still played a unique and significant direct role in the prediction of anxiety symptoms, but had no indirect effect via the mediators of worry and rumination. Additionally, adding symptoms of depression to the analysis as a covariate yielded comparable results.

\section{Symptoms of Depression}

The next model examined whether worry and rumination mediated the relation between neuroticism and depressive symptoms. Results revealed a significant relation between neuroticism and depression $(B=.20, \mathrm{SE}=.02, p<.001)$. Neuroticism, worry and rumination together explained $45 \%$ of the variance in depressive symptoms. As shown in Fig. 2, 74\% of this variance was explained by the direct relation between neuroticism and depression and this direct relation was significant $(B=.14, \mathrm{SE}=.02, p<.001)$. The remaining $26 \%$ was accounted for by the total indirect effect of the mediators worry and rumination, which was also found to be significant [95\% CI (.02-.09)]. A significant proportion of $18 \%$ was accounted for by worry [95\% CI (.00-.07)], whereas only $8 \%$ was explained by rumination, an effect which was not significant $[95 \%$ CI (-.01.04)]. These findings indicate that part of the relation between neuroticism and depressive symptoms was mediated by worry.

However, when adding anxiety symptoms as a covariate to this model, a somewhat different picture emerged. Although the relation between neuroticism and depression $(B=.11, \mathrm{SE}=.02, p<.001)$ was still significant and neuroticism, worry and rumination together explained $50 \%$ of the variance in depressive symptoms (when controlling

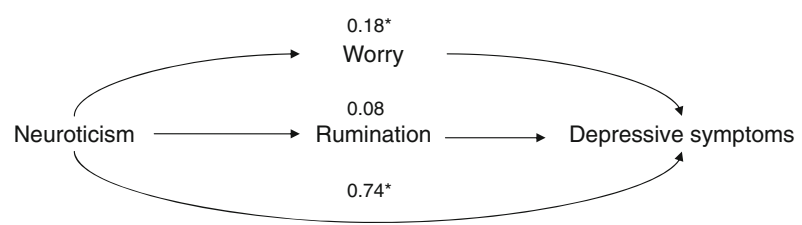

Fig. 2 Mediation effects of rumination and worry on the relation between neuroticism and depressive symptoms. Note. Numbers in the figure are percentages of explained variance in depressive symptoms. * Path significant at $p<.01$ 
for anxiety symptoms), now $98 \%$ of this variance was explained by the direct relation between neuroticism and depression and this direct relation was significant $(B=.11$, $\mathrm{SE}=.03, p<.001)$. The remaining $2 \%$ was accounted for by the total indirect effect of the mediators worry and rumination, which appeared to be non-significant [95\% CI $(-.01-.02)]$ and the same was true for the unique indirect effects of worry $[95 \% \mathrm{CI}(-.01-.01)]$ and rumination $[95 \%$ CI (-.01-.01)]. These findings indicate the relation between neuroticism and depressive symptoms was not mediated by worry or rumination when controlling for anxiety symptoms.

Additionally, a model including behavioral inhibition instead of neuroticism as independent variable was also tested to examine whether behavioral inhibition also plays a role in other types of psychopathology such as depression. This analysis indicated that there was no significant relation between behavioral inhibition and depression when controlling for neuroticism $(B=.06, \mathrm{SE}=.01$, $p<.001)$. Moreover, behavioral inhibition, worry, and rumination together explained only $18 \%$ of the variance in depression scores when controlling for neuroticism. Of the total effect, $38 \%$ of the variance could be explained by the indirect effect through worry and rumination [95\% CI (.02$.11)]$, with worry accounting for $26 \%$ of this explained variance [95\% CI (.01-.09)] and rumination for 12\% [95\% CI (-.01-.07)], with the latter effect being non-significant. These findings indicate that when controlling for neuroticism, the temperamental trait of behavioral inhibition did not play a unique direct role in the prediction of depression symptoms, but there was a significant indirect effect of behavioral inhibition on depressive symptoms via the mediator of worry. When adding anxiety symptoms as a covariate to this model the relation between behavioral inhibition and depression was no longer significant $(B=.01, \mathrm{SE}=.01, p=.30)$ and neither behavioral inhibition $(B=.01, \mathrm{SE}=.01, p=.30,98 \%$ of the explained variance) nor worry [95\% CI (-.003-.002)] and rumination [95\% CI (-.003-.001)] were significantly associated with depressive symptoms. These findings point out that when controlling for neuroticism and anxiety symptoms, the temperamental trait of behavioral inhibition did not play a role in the prediction of depression symptoms.

\section{Sleep Difficulties}

Results indicated that there was a significant relation between neuroticism and sleep difficulties $(B=.59$, $\mathrm{SE}=.08, p<.001)$. Neuroticism, worry, and rumination together accounted for $27 \%$ of the variance in sleep difficulties (see Fig. 3). Eighty-six percent of this explained variance consisted of the direct pathway between

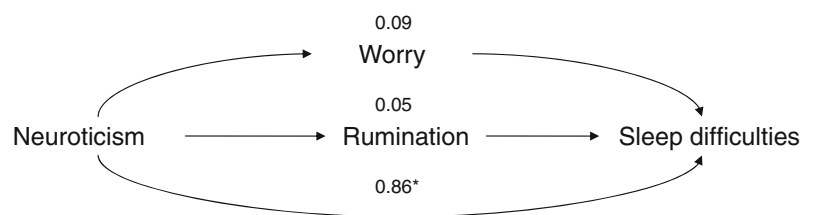

Fig. 3 Mediation effects of rumination and worry on the relation between neuroticism and sleep difficulties. Note. Numbers in the figure are percentages of explained variance in sleep difficulties. * Path significant at $p<.01$

neuroticism and sleep difficulties. This direct relation was significant $(B=.51, \mathrm{SE}=.10, p<.001)$. The remaining $14 \%$ was explained by the indirect path via worry $(9 \%)$ and rumination $(5 \%)$. However, this total indirect effect was not significant [95\% CI (-.05-.21)], and this was also true for the specific indirect effects of worry [95\% CI $(-.09-$ .19)] and rumination [95\% CI (-.08-.13)]. Thus, neither worry nor rumination emerged as mediators in the relationship between neuroticism and sleep difficulties.

Further, a model including behavioral inhibition instead of neuroticism as independent variable was also tested for sleeping difficulties. Results indicated that there was no significant relation between behavioral inhibition and sleep difficulties. Behavioral inhibition, worry, and rumination together accounted for $18 \%$ of the variance in sleep difficulties. Sixty-two percent of this explained variance consisted of the direct pathway between behavioral inhibition and sleep difficulties, whereas the remaining $38 \%$ was explained by the indirect path via worry $(26 \%)$ and rumination (12\%). Although the direct effect of behavioral inhibition on sleep difficulties was not significant, the total indirect effect was significant [95\% CI (.02-.11)], and this was also the case for the specific indirect effect of worry [95\% CI (.01-.09)] but not for rumination [95\% CI (-.01.07)]. However, when controlling for neuroticism in this model, no significant direct effect of behavioral inhibition or indirect effect of worry or rumination on sleep difficulties was found, only a significant partial effect of neuroticism on sleep difficulties $(B=.47, \mathrm{SE}=.11, p<$ .001). These findings indicate that when controlling for neuroticism, the temperamental trait of behavioral inhibition did not have a unique direct effect or an indirect effect via worry and/or rumination on sleep difficulties.

Additionally, as sleep difficulties in children may arise as a consequence of symptoms of anxiety and depression, or may predispose children to symptoms of psychopathology, further models were examined in which these ideas were statistically tested. First, a mediation model was tested in which symptoms of anxiety and depression mediated the link between neuroticism and sleep difficulties. As noted previously, the relation between neuroticism and sleep difficulties was significant $(B=.59, \mathrm{SE}=.08$, 
$p<.001)$. In this model, neuroticism, anxiety and depression together explained $37 \%$ of the variance in sleep difficulties scores. Thirty-six percent of this explained variance ran through the direct pathway between neuroticism and sleep difficulties, but this relation was not significant $(B=.21, \mathrm{SE}=.11, p=.05)$. The remaining $64 \%$ of the variance in sleep difficulties scores was explained by the indirect pathway via depression and anxiety. The total indirect effect was significant [95\% CI (.22-.57)] and this was also true for the specific indirect effects via depression [95\% CI (.06-.32)] and anxiety [95\% CI (.05-.34)]. Thirtyfour percent of the variance was accounted for by the indirect effect via depression, whereas $30 \%$ was explained by the indirect effect via anxiety. These findings indicate that symptoms of anxiety and depression mediated the relation between neuroticism and sleep difficulties.

Second, two models were tested in which sleep difficulties mediated the link between neuroticism and symptoms of depression and anxiety. In the first model, which included symptoms of anxiety, the relation between neuroticism and anxiety symptoms was significant $(B=.61$, $\mathrm{SE}=.08, p<.001)$ and neuroticism and sleep difficulties together explained $48 \%$ of the variance in anxiety scores. Seventy-nine percent of this explained variance ran through the direct pathway between neuroticism and anxiety problems $(B=.78, \mathrm{SE}=.07, p<.001)$, whereas the remaining $21 \%$ of the variance was explained by the indirect pathway via sleep difficulties. This indirect effect was significant [95\% CI (.08-.29)]. Thus, sleep difficulties emerged as a (partial) mediator of the relation between neuroticism and anxiety problems.

The second model included symptoms of depression instead of anxiety as outcome measure. Results indicated that neuroticism and sleep difficulties together accounted for $47 \%$ of the variance in depressive symptoms. Seventysix percent of this explained variance consisted of the direct pathway between neuroticism and depression, whereas the remaining $24 \%$ was explained by the indirect path via sleep difficulties. The direct effect $(B=.15$, $\mathrm{SE}=.02, p<.001)$ and the indirect effect $[95 \% \mathrm{CI}(.02-$ .08)] were both significant. That is, sleep problems emerged as a (partial) mediator of the relation between neuroticism and depressive symptoms.

\section{Discussion}

The present study examined the role of worry and rumination as indicators of repetitive negative thoughts in the vulnerability for emotional problems in non-clinical children aged 8-13 years. The main results can be catalogued as follows. To begin with, as hypothesized, significant positive correlations between measures of general vulnerability (neuroticism), repetitive negative thoughts (worry and rumination), and various types of emotional symptoms (anxiety, depression, and sleep difficulties) were found. Further and most importantly, support was found for the hypothesized model in which worry and rumination partly mediated the relation between neuroticism and symptoms of anxiety and depression. This finding is not only in line with the notion that repetitive negative thoughts are involved in the pathogenesis of emotional symptoms in children (see for reviews Abela and Hankin 2007; Muris 2007), but also indicates that worry and rumination in young people seem to play a similar role in the vulnerability for such problems as in adults (Muris et al. 2005b; Roelofs et al. 2008).

The personality trait of neuroticism has been widely acknowledged as a general vulnerability factor that makes individuals prone to exhibit symptoms of psychopathology (e.g., Eysenck and Eysenck 1985), and the current data indicate that this also appears to be the case in youths (see for reviews Muris and Ollendick 2005; Nigg 2006). Meanwhile, several authors have criticized this notion by pointing out that neuroticism is in fact "a non-informative marker of vulnerability to psychopathology", because it is largely unknown what underlying mechanisms cause the high symptom levels of individuals who display the typical features of this trait (Ormel et al. 2004, p. 906). As such, it is of interest to note that researchers have begun to examine whether vulnerability for emotional problems can be better perceived in terms of a hierarchical model in which the general vulnerability factor of neuroticism is associated with a variety of specific vulnerability markers, which in turn determine the specific categories of psychopathology (e.g., Norton and Mehta 2007; Sexton et al. 2003). In line with this, the model described by Muris et al. (2005b) proposes that repetitive negative thoughts should be seen as a cognitive concomitant of neuroticism (cf. Segerstrom et al. 2000), which predisposes individuals to develop symptoms of anxiety and depression. To the present authors' knowledge, the present data are the first to support that such a hierarchical model of the vulnerability for emotional problems also applies to youths. Obviously, more research is needed to investigate other variables (e.g., anxiety sensitivity, intolerance of uncertainty, meta-cognition; see Norton and Mehta 2007; Sexton et al. 2003) that may act as mediators in the link between general vulnerability (i.e., neuroticism) and various types of internalizing problems in children and adolescents.

The hypothesized mediation model "neuroticism $\rightarrow$ worry/rumination $\rightarrow$ emotional problems" did not apply to children's sleep problems. More precisely, although positive and significant correlations were observed among scales for measuring neuroticism, worry/rumination, and sleep difficulties, no evidence was obtained for the idea 
that indices of repetitive negative thoughts acted as an intermediate variable in the link between general vulnerability and such problems. Thus, while it seems intuitively plausible that children who display high levels of worry and rumination also exhibit certain troublesome sleep behaviors (e.g., problems with falling asleep), sleep difficulties were mainly explained by the direct path of neuroticism and not by the indirect path via the cognitive mediators worry and rumination. Several explanations may account for this result. First, it should be noted that the index that was used in this study for assessing sleep difficulties (i.e., the SSR) might have been too broad as it also measures a variety of problematic sleep behaviors (e.g., nightmares, fighting with parents about going to bed, falling asleep in the same bed every night) that seem to be less susceptible to repetitive negative thoughts. Second, there might be another mechanism that accounts for the specific vulnerability to develop sleep difficulties. One candidate may be low vagal activity, which is known as a physiological marker of vulnerability that is associated with poor stress regulation (Porges 1992). There is research showing that a low vagal tone is associated with the personality trait of neuroticism (Haug et al. 1994) and also appears to be related to children's sleep problems (ElSheikh et al. 2007). As such, it would be interesting to examine a mediation model in which low vagal activity acts as a mediator between the general vulnerability of neuroticism and sleep difficulties. Third and finally, it is also possible that sleep difficulties do not represent a unique psychopathological entity, but rather should be regarded as an additional symptom of other emotional problems or the other way around may predispose children to symptoms of psychopathology. Additionally, these ideas were also statistically tested in this study. Analyses yielded a model in which anxiety and depression mediated the relation between neuroticism and sleep difficulties, whereas support was also found for models in which sleep problems partly mediated the relation between neuroticism and symptoms of anxiety and depression.

However, due to the cross-sectional nature of this study, no cause-effect relations could be established, and therefore it remains unclear whether sleep difficulties in children can best be seen as an epiphenomenon of high levels of anxiety or depression (Lichstein 2006), or may predispose children to symptoms of psychopathology (Fallone et al. 2002; Harvey 2001a, b), or even whether there is a reciprocal relation between these concepts in which sleep problems and daytime sleepiness enhance the risk of developing anxiety and depressive symptoms, whereas these symptoms in turn also make children more vulnerable for sleep difficulties. Longitudinal studies are needed to address this issue. Moreover, the current study only assessed a limited number of relevant variables. In reality the relation between sleep difficulties and emotional problems in youths might be far more complicated, and involves various biological, psychological and social processes (Dahl and Lewin 2002).

Previous data also confirmed the notion that behavioral inhibition represents a vulnerability factor for anxiety problems in youths (Fox et al. 2005). As hypothesized, even when controlling for neuroticism, this temperament factor still accounted for a significant and unique proportion of the variance in children's anxiety scores (see also Muris et al. 2009a). Behavioral inhibition did not account for a significant proportion of the variance in children's depression or sleep disturbances scores when controlling for neuroticism. Thus, it seems that behavioral inhibition is a unique vulnerability factor for anxiety problems and not for depression or sleep disturbances. Interestingly, the results also indicated that while the relation between neuroticism/behavioral inhibition and anxiety symptoms was partly mediated by repetitive negative thoughts, behavioral inhibition only had a direct effect on anxiety symptoms once controlling for neuroticism. These findings can be explained by bearing in mind that behavioral inhibition can best be seen as a mixture of the personality traits of neuroticism and extraversion (Muris and Dietvorst 2006; Muris et al. 2009a; Shatz 2005). Thus, the part of behavioral inhibition that overlaps with neuroticism seems to partially exert its influence on anxiety via the mediators of worry and rumination, whereas the part mapping on extraversion might influence these symptoms via another mechanism (e.g., avoidant coping). Further studies are needed to further elucidate the role of neuroticism, extraversion, and behavioral inhibition in anxiety pathology, and the precise mechanisms by which these general vulnerability factors operate.

In line with previous research in children (Muris et al. 2004, 2009b) and adults (Fresco et al. 2002; Muris et al. 2005b; Roelofs et al. 2008; Segerstrom et al. 2000), the present results demonstrated that (1) worry and rumination were closely related to each other, and (2) both types of repetitive negative thoughts were convincingly associated with symptoms of both anxiety and depression. Meanwhile, the mediation analyses examining worry and rumination as specific vulnerability factors revealed that worry and rumination both made a significant contribution to the model for anxiety symptoms, and that this was also true when controlling for depressive symptoms. Worry only declared a significant proportion of the variance in the model for depressive symptoms. However, when controlling for anxiety symptoms none of the mediators made a significant contribution to the model. While the findings of the analyses correspond nicely with those obtained by Muris et al. (2004, 2009b), they are certainly not in agreement with the original idea that worry would be more 
relevant for anxiety, whereas rumination would be more pertinent to depression. It may well be that sample differences account for this pattern of results. That is, the present study as well as previous investigations by Muris et al. $(2004,2009 b)$ relied on non-clinical youths, for which it may well be the case that worry is a more common phenomenon than rumination. However, research by Roelofs et al. (2008) demonstrated that in a sample of clinically depressed patients, rumination emerged as the main predictor of anxiety and depressive symptoms, in spite of the fact that worry was also linked to both types of emotional symptoms. For these patients, rumination may have been a more recognizable type of repetitive negative thought as compared to worry. Future studies examining the relative contributions of worry and rumination to various emotional problems in clinical and non-clinical adult and child populations might further clarify this issue.

The results from the present study may have implications for clinical practice. Although it must be kept in mind that this study was cross-sectional in nature, the current findings indicate that repetitive negative thoughts could play a role in anxiety and depression. This would underline the importance of cognitive treatment approaches directed at correcting and modifying these cognitive factors. The treatment of anxiety and depressive may focus on strategies to diminish the tendency to worry or ruminate. One recent potential treatment to change these tendencies might be mindfulness-based cognitive therapy for depression (Segal et al. 2002) which might be suitable for youths as well. This therapy aims to let participants focus on the present and to observe their (repetitive negative) thoughts instead of dwelling on them. Preliminary evidence shows that, at least in adults, a mindfulness-based cognitive therapy specifically focused on reducing ruminative thoughts was effective in reducing rumination and depressive symptoms in medication-refractory depression (Watkins et al. 2007).

It should be acknowledged that the present study suffers from various limitations. First, this research was correlational in nature, and hence it is not possible to draw conclusions on cause-effect relationships between neuroticism, repetitive negative thought, and emotional problems. However, there are good reasons to believe that neuroticism acts as a precursor rather than as a consequence of child psychopathology (see Muris and Ollendick 2005) and current theories in clinical psychology assume that neuroticism is a higher-order vulnerability factor, whereas repetitive negative thoughts can better be qualified as a second-order vulnerability factor (Norton et al. 2005; Sexton et al. 2003). Second, as mentioned earlier, the study merely included non-clinical subjects. In addition, no diagnostic instrument was employed to identify children who really suffered from anxiety, depressive, and sleeping disorders, and therefore it remains unclear to what extent these findings can be generalized to samples of clinically diagnosed youths. Also our sample size was too restricted to test separate models for boys and girls. However, girls in our sample did tend to worry and ruminate more than boys and previous research also showed that girls are more prone to develop a depressive or an anxiety disorder than boys (e.g., Craske 2003; Lewinsohn et al. 1994). Therefore, it would be interesting for future research to test separate mediational models for boys and girls to study whether differences in repetitive negative thought play a role in the gender difference in prevalence of depressive and anxiety symptoms and disorders. Third, results of this study should be interpreted with some caution due to confounding of measures. That is, some of the items of the anxiety scale explicitly referred to worry, the depression subscale included an item about tiredness, the rumination subscale contained items that refer to symptoms of depression, whereas repetitive negative thought, and more specific worry are a prominent feature of generalized anxiety disorders. Further, this investigation solely relied on children's self-report and only employed one instrument for measuring each of the constructs. Future studies should use a multi-trait, multi-method approach to get around this problem and include parent- and/or teacher rating, and objective measures of sleep such as actigraphy data next to children's self-reports. Fourth and finally, it is also important to note that the vulnerability models that were tested in this research are a simplification of the reality. Developmental psychopathology accounts propose that emotional problems in youths are the result from multiple factors, reflecting risk as well as resilience, that operate in dynamic interaction (Cicchetti and Cohen 1995), and hence research that includes other relevant variables would be especially welcome.

In spite of these limitations, the current findings seem to indicate that worry and rumination contribute to children's vulnerability for anxiety and depression. As it is increasingly acknowledged that proneness to internalizing psychopathology becomes particularly manifest when youths are exposed to stressful life events (e.g., Brozina and Abela 2006; Morris et al. 2008), it might be also interesting for future studies to prospectively examine the role of repetitive negative thoughts in the vulnerability for emotional problems within a diathesisstress context.

Acknowledgments Parents, children and teachers from primary schools "Smitshoek" in Barendrecht and "De Bron", "De Notenbalk" en "De Kim" in Zwijndrecht are kindly thanked for their participation in the present study. 
Open Access This article is distributed under the terms of the Creative Commons Attribution Noncommercial License which permits any noncommercial use, distribution, and reproduction in any medium, provided the original author(s) and source are credited.

\section{References}

Abela, J. R. Z., Brozina, K., \& Haigh, E. P. (2002). An examination of the response styles theory of depression in third- and seventhgrade children: A short-term longitudinal study. Journal of Abnormal Child Psychology, 30, 515-527.

Abela, J. R. Z., \& Hankin, B. L. (2007). Childhood and adolescent depression: Causes, treatment, and prevention. New York: Guilford Press.

Alfano, C. A., Ginsburg, G. S., \& Kingery, J. N. (2007). Sleep-related problems among children and adolescents with anxiety disorders. Journal of American Academy of Child and Adolescent Psychiatry, 46, 224-232.

American Psychiatric Association. (2000). Diagnostic and statistical manual of mental disorders (4th edition text revision ed.). Washington, DC: American Psychiatric Association.

Barbaranelli, C., Caprara, G. V., Rabasca, A., \& Pastorelli, C. (2003). A questionnaire for measuring the Big Five in late childhood. Personality and Individual Differences, 34, 645-664.

Biederman, J., Hirshfeld-Becker, D. R., Rosenbaum, J. F., Friedman, D., Snidman, N., Kagan, J., et al. (2001). Further evidence of the association between behavioral inhibition and social anxiety in children. American Journal of Psychiatry, 158, 1673-1679.

Biederman, J., Rosenbaum, J. F., Bolduc-Murphy, E. A., Faraone, S. V., Chaloff, J., Hirshfeld, D. R., et al. (1993). A 3-year follow-up of children with and without behavioral inhibition. Journal of the American Academy of Child and Adolescent Psychiatry, 32(4), 814-821.

Bishop, G., Spence, S. H., \& McDonald, C. (2003). Can parents and teachers provide a reliable and valid report of behavioral inhibition? Child Development, 74, 1899-1917.

Borkovec, T. D., Robinson, E., Pruzinsky, T., \& DePree, J. A. (1983). Preliminary exploration of worry: Some characteristics and processes. Behaviour Research and Therapy, 21, 9-16.

Brozina, K., \& Abela, J. R. Z. (2006). Behavioural inhibition, anxious symptoms, and depressive symptoms: A short-term prospective examination of a diathesis-stress model. Behaviour Research and Therapy, 44, 1337-1346.

Carney, C., Edinger, J., Meyer, B., Lindman, L., \& Istre, T. (2006). Symptom-focused rumination and sleep-disturbance. Behavioral Sleep Medicine, 4, 228-241.

Chorpita, B. F., Tracey, S. A., Brown, T. A., Collica, T. J., \& Barlow, D. H. (1997). Assessment of worry in children and adolescents: An adaptation of the Penn State Worry Questionnaire. Behaviour Research and Therapy, 35, 569-581.

Chorpita, B. F., Yim, L., Moffit, C., Umemoto, L. A., \& Francis, S. E. (2000). Assessment of symptoms of DSM-IV anxiety and depression in children: A revised child anxiety and depression scale. Behaviour Research and Therapy, 38, 835-855.

Cicchetti, D., \& Cohen, D. J. (1995). Perspectives on developmental psychopathology. In D. Cicchetti \& D. J. Cohen (Eds.), Developmental psychopathology: Volume 1. Theory and methods (pp. 3-20). New York: Wiley.

Costello, E. J., Mustillo, S., Erkanli, A., Keeler, G., \& Angold, A. (2003). Prevalence and development of psychiatric disorders in childhood and adolescence. Archives of General Psychiatry, 60 , 837-844.
Craske, M. G. (1997). Fear and anxiety in children and adolescents. Bulletin of the Menninger Clinic, 61(2, Suppl A), 4-36.

Craske, M. G. (2003). Origins of phobias and anxiety disorders. Why more women than men?. Oxford: Elsevier.

Dahl, R. E., \& Lewin, D. S. (2002). Pathways to adolescent health: Sleep regulation and behavior. Journal of Adolescent Health, 31, $175-184$.

Davey, G. C. L. (1994). Worrying, social problem solving abilities, and problem-solving confidence. Behaviour Research and Therapy, 32, 327-330.

Edwards, S. L. (2007). Temperament and environmental risk factors contributing to anxiety symptoms in preschool-aged children. Unpublished Ph.D. thesis, Macquarie University, Australia.

El-Sheikh, M., Erath, S. A., \& Keller, P. S. (2007). Children's sleep and adjustment: The moderating role of vagal regulation. Journal of Sleep Research, 16, 396-405.

Eysenck, H. J. (1967). The biological basis of personality. Springfield, IL: Charles C. Thomas.

Eysenck, H. J., \& Eysenck, M. W. (1985). Personality and individual differences. New York: Plenum Press.

Fallone, G., Owens, J. A., \& Deane, J. (2002). Sleepiness in children and adolescents: Clinical implications. Sleep Medicine Reviews, 6, 287-306.

Fleming, J. E., \& Offord, D. R. (1990). Epidemiology of childhood depressive disorders: A critical review. Journal of the American Academy of Child and Adolescent Psychiatry, 29, 571-580.

Fox, N. A., Henderson, H. A., Marshall, P. J., Nichols, K. E., \& Ghera, M. M. (2005). Behavioral inhibition: Linking biology and behavior within a developmental framework. Annual Review of Psychology, 56, 235-262.

Fresco, D. M., Frankel, A. N., Mennin, D. S., Turk, C. L., \& Heimberg, R. G. (2002). Distinct and overlapping features of rumination and worry: The relationship of cognitive production to negative affective states. Cognitive Therapy and Research, 26, $179-188$.

Guastella, A. J., \& Moulds, M. L. (2007). The impact of rumination on sleep quality following a stressful life event. Personality and Individual Differences, 42, 1151-1162.

Harvey, A. G. (2000). Pre-sleep cognitive activity: A comparison between sleep-onset insomniacs and good sleepers. British Journal of Clinical Psychology, 39, 275-286.

Harvey, A. G. (2001a). I can't sleep, my mind is racing! An investigation of strategies of thought control in insomnia. Behavioural and Cognitive Psychotherapy, 29, 3-11.

Harvey, A. G. (2001b). Insomnia: Symptoms or diagnosis? Clinical Psychology Review, 21, 1037-1059.

Haug, T. T., Svebak, S., Hausken, T., Wilhemsen, I., Berstad, A., \& Ursin, H. (1994). Low vagal activity as mediating mechanisms for the relationship between personality factors and gastric symptoms in functional dyspepsia. Psychosomatic Medicine, 56, 181-186.

Hong, R. Y. (2007). Differential associations with anxious and depressive symptoms and coping behavior. Behaviour Research and Therapy, 45, 277-290.

Kagan, J. (1994). Galen's prophecy. New York, NY: Basic Books.

Kirkegaard Thomsen, D., Mehlsen, M., Christensen, S., \& Zachariae, R. (2003). Rumination-Relationship with negative mood and sleep quality. Personality and Individual Differences, 34, 1293-1301.

Lewinsohn, P. M., Clarke, G. N., Seeley, J. R., \& Rohde, P. (1994) Major depression in community adolescents: Age at onset, episode duration, and time to recurrence. Journal of the American Academy of Child and Adolescent Psychiatry, 33, 809-818.

Lichstein, K. L. (2006). Secondary insomnia: A myth dismissed. Sleep Medicine Reviews, 10, 3-5. 
Liu, X., Buysse, D. J., Gentzler, A. L., Kiss, E., Mayer, L., Kapornai, K., et al. (2007). Insomnia and hypersomnia associated with depressive phenomenology and comorbidity in childhood depression. Sleep, 30, 83-90.

Lonigan, C. J., \& Phillips, B. M. (2001). Temperamental influences on the development of anxiety disorders. In M. W. Vasey \& M. R. Dadds (Eds.), The developmental psychopathology of anxiety (pp. 60-91). New York: Oxford University Press.

McLaughlin, K., Sibrava, N., Behar, E., \& Borkovec, T. D. (2006). Recurrent negative thinking in emotional disorders: Worry, depressive rumination, and trauma recall. In S. Sassaroli, G. Rugerio, \& R. Lorenzini (Eds.), Worry need of control, and other core cognitive constructs in anxiety and eating disorders (pp. 37-67). Milan, Italy: Raphael Cortina.

Morris, M. C., Ciesla, J. A., \& Garber, J. (2008). A prospective study of the cognitive-stress model of depressive symptoms in adolescents. Journal of Abnormal Psychology, 117, 719-734.

Muris, P. (2007). Normal and abnormal fear and anxiety in children and adolescents. Oxford: Elsevier.

Muris, P., Bos, A. E. R., Mayer, B., Verkade, R., Thewissen, V., \& Dell'Avvento, V. (2009a). Relations among behavioral inhibition, Big Five personality factors, and anxiety disorder symptoms in non-clinical children. Personality and Individual Differences, 46, 525-529.

Muris, P., \& Dietvorst, R. (2006). Underlying personality characteristics of behavioral inhibition in children. Child Psychiatry and Human Development, 36, 437-445.

Muris, P., Fokke, M., \& Kwik, D. (2009b). The ruminative response style in adolescents: An examination of its specific link to symptoms of depression. Cognitive Therapy and Research, 33, 21-32.

Muris, P., Meesters, C., \& Diederen, R. (2005a). Psychometric properties of the Big Five Questionnaire for Children (BFQ-C) in a Dutch sample of young adolescents. Personality and Individual Differences, 38, 1757-1769.

Muris, P., Meesters, C., \& Schouten, E. (2002). A brief questionnaire of DSM-IV defined anxiety and depression symptoms among children. Clinical Psychology and Psychotherapy, 9, 430442.

Muris, P., Merckelbach, H., Schmidt, H., Gadet, B., \& Bogie, N. (2001). Anxiety and depression as correlates of self-reported behavioural inhibition in normal adolescents. Behaviour Research and Therapy, 39, 1051-1061.

Muris, P., Merckelbach, H., Wessel, I., \& van de Ven, M. (1999). Psychopathological correlates of self-reported behavioural inhibition in normal children. Behaviour Research and Therapy, 37, $575-584$

Muris, P., \& Ollendick, T. H. (2005). The role of temperament in the etiology of child psychopathology. Clinical Child and Family Psychology Review, 8, 271-289.

Muris, P., Roelofs, J., Meesters, C., \& Boomsma, P. (2004). Rumination and worry in non-clinical adolescents. Cognitive Therapy and Research, 28, 539-554.

Muris, P., Roelofs, J., Rassin, E., Franken, I., \& Mayer, B. (2005b). Mediating effects of rumination and worry on the links between neuroticism, anxiety, and depression. Personality and Individual Differences, 39, 1105-1111.

Nigg, J. T. (2006). Temperament and developmental psychopathology. Journal of Child Psychology and Psychiatry, 47, 395-422.

Nolen-Hoeksema, S. (1998). Ruminative coping with depression. In J. Heckhausen \& C. S. Dweck (Eds.), Motivation and selfregulation across the life span (pp. 237-256). New York: Cambridge University Press.

Nolen-Hoeksema, S. (2000). The role of rumination in depressive disorders and mixed anxiety/depressive symptoms. Journal of Abnormal Psychology, 109, 504-511.
Nolen-Hoeksema, S., Wisco, B. E., \& Lyubomirsky, S. (2008). Rethinking rumination. Perspectives on Psychological Science, $3,400-424$.

Norton, P. J., \& Mehta, P. D. (2007). Hierarchical model of vulnerabilities for emotional disorders. Cognitive Behavior Therapy, 36, 240-254.

Norton, P. J., Sexton, K. A., Walker, J. R., \& Norton, G. R. (2005). Hierarchical model of vulnerabilities for anxiety: Replication and extension with a clinical sample. Cognitive Behaviour Therapy, 34, 50-63.

Ormel, J., Rosmalen, J., \& Farmer, A. (2004). Neuroticism: A noninformative marker of vulnerability to psychopathology. Social Psychiatry and Psychiatric Epidemiology, 39, 906-912.

Owens, J. A., Maxim, R., Nobile, C., McGuinn, M., \& Msall, M. (2000). Parental and self-report of sleep in children with attention-deficit/hyperactivity disorder. Archives of Pediatrics and Adolescent Medicine, 154, 549-555.

Porges, S. W. (1992). Vagal tone: A physiologic marker of stress vulnerability. Pediatrics, 90, 498-504.

Preacher, K. J., \& Hayes, A. F. (2008). Asymptotic and resampling strategies for assessing and comparing indirect effects in multiple mediator models. Behaviour Research Methods, 40, 879-891.

Prior, M., Sanson, A., \& Oberklaid, F. (1989). The Australian temperament project. In D. Kohnstamm, J. Bates, \& M. Rothbart (Eds.), Temperament in childhood (pp. 537-554). Chichester, UK: Wiley.

Roelofs, J., Huibers, M., Peeters, F., Arntz, A., \& van Os, J. (2008). Rumination and worrying as possible mediators in the relation between neuroticism and symptoms of depression and anxiety in clinically depressed individuals. Behaviour Research and Therapy, 46, 1283-1289.

Rood, L., Roelofs, J., Bögels, S. M., Nolen-Hoeksema, S., \& Schouten, E. (2009). The influence of emotion-focused rumination and distraction on depressive symptoms in non-clinical youth: A metaanalytic review. Clinical Psychology Review, 29, 607-616.

Ryan, D. N., Puig-Antich, J., Rabinovich, H., Robinson, D., Ambrosini, P. J., Nelson, B., et al. (1987). The clinical picture of major depression in children and adolescents. Archives of General Psychiatry, 44, 854-861.

Segal, Z. V., Williams, J. M. C., \& Teasdale, J. D. (2002). Mindfulness-based cognitive therapy for depression-A new approach to preventing relapse. New York: Guilford Press.

Segerstrom, S. C., Tsao, J. C. I., Alden, L. I., \& Craske, M. G. (2000). Worry and rumination: Repetitive thoughts as a concomitant and a predictor of negative mood. Cognitive Therapy and Research, 24, 671-688.

Sexton, K. A., Norton, P. J., Walker, J. R., \& Norton, G. R. (2003). Hierarchical model of generalized and specific vulnerabilities in anxiety. Cognitive Behavior Therapy, 32, 82-94.

Shatz, S. M. (2005). The psychometric properties of the Behavioral Inhibition Scale in a college-aged sample. Personality and Individual Differences, 39, 331-339.

Silverman, W. K., La Greca, A. M., \& Wasserstein, S. (1995). What do children worry about? Worries and their relation to anxiety. Child Development, 66, 671-686.

Sobel, M. E. (1986). Some new results on indirect effects and their standard errors in covariance structure models. In N. Tuma (Ed.), Sociological methodology 1986 (pp. 159-186). Washington: American Sociological Association.

Starcevic, V. (1995). Pathological worry in major depression: A preliminary report. Behaviour Research and Therapy, 33, 55-56.

Strauss, C. C., Frame, C. L., \& Forehand, R. (1987). Psychosocial impairment associated with anxiety in children. Journal of Clinical Child and Adolescent Psychology, 16, 235-239. 
Van Brakel, A. M. L., \& Muris, P. (2006). A Brief Scale for Measuring "Behavioral Inhibition to the Unfamiliar" in Children. Journal of Psychopathology and Behavioral Assessment, 28, 79-84.

Watkins, E., \& Moulds, M. (2005). Distinct modes of ruminative selffocus: Impact of abstract versus concrete rumination on problem solving in depression. Emotion, 5, 319-328.

Watkins, E., Scott, J., Wingrove, J., Rimes, K., Bathurst, N., Steiner, H., et al. (2007). Rumination-focused cognitive behaviour therapy for residual depression: A case series. Behaviour Research and Therapy, 45, 2144-2154.
Watkins, E., \& Teasdale, J. D. (2001). Rumination and an overgeneral memory in depression: Effects on self-focus and analytic thinking. Journal of Abnormal Psychology, 110, 353-357.

Wicklow, A., \& Espie, C. A. (2000). Intrusive thoughts and their relationship to actigraphic measurement of sleep: Towards a cognitive model of insomnia. Behavioural Research and Therapy, 38, 679-693.

Ziegert, D. I., \& Kistner, J. A. (2002). Response styles theory: Downward extension to children. Journal of Clinical Child and Adolescent Psychology, 31, 325-334. 